\title{
The Epigenetic Factor KDM2B Regulates EMT and Small GTPases in Colon Tumor Cells
}

\author{
Nefeli Zacharopoulou ${ }^{a}$ Anna Tsapara ${ }^{a} \quad$ Galatea Kallergia Evi Schmid ${ }^{b}$ \\ Saad Alkahtania,c Saud Alarific Philip N. Tsichlis ${ }^{d}$ Sotirios C. Kampranis ${ }^{a}$ \\ Christos Stournaras ${ }^{a}$ \\ aDepartment of Biochemistry, University of Crete Medical School, Voutes, Heraklion, Greece, \\ ${ }^{b}$ Department of Pediatric Surgery \& Pediatric Urology, Children's Hospital, Eberhard-Karls-University \\ Tuebingen, Tuebingen, Germany, 'Department of Zoology, Science College, King Saud University, \\ Riyadh, Saudi Arabia, ${ }^{d}$ Molecular Oncology Research Institute, Tufts Medical Center, Boston, USA
}

\section{Keywords}

KDM2B • Small-GTPases • EMT • Colon cancer

\begin{abstract}
Background/Aims: The epigenetic factor KDM2B is a histone demethylase expressed in various tumors. Recently, we have shown that KDM2B regulates actin cytoskeleton organization, small Rho GTPases signaling, cell-cell adhesion and migration of prostate tumor cells. In the present study, we addressed its role in regulating EMT and small GTPases expression in colon tumor cells. Methods: We used RT-PCR for the transcriptional analysis of various genes, Western blotting for the assessment of protein expression and immunofluorescence microscopy for visualization of fluorescently labeled proteins. Results: We report here that KDM2B regulates EZH2 and BMI1 in HCT116 colon tumor cells. Knockdown of this epigenetic factor induced potent up-regulation of the protein levels of the epithelial markers E-cadherin and ZO-1, while the mesenchymal marker $\mathrm{N}$-cadherin was downregulated. On the other hand, KDM2B overexpression downregulated the levels of both epithelial markers and upregulated the mesenchymal marker, suggesting control of EMT by KDM2B. In addition, RhoA, RhoB and RhoC protein levels diminished upon KDM2B-knockdown, while all three small GTPases became upregulated in KDM2B-overexpressing HCT116 cell clones. Interestingly, Rac1 GTPase level increased upon KDM2B-knockdown and diminished in KDM2B-overexpressing HCT116 colon tumor- and DU-145 prostate cancer cells. Conclusions: These results establish a clear functional role of the epigenetic factor KDM2B in the regulation of EMT and small-GTPases expression in colon tumor cells and further support the recently postulated oncogenic role of this histone demethylase in various tumors.

S. C. Kampranis and C. Stournaras contributed equally to this work.

Prof. Dr. Christos Stournaras

Department of Biochemistry,

University of Crete Medical School, 71110 Heraklion (Greece)

E-Mail stournac@uoc.gr 


\section{Cellular Physiology Cell Physiol Biochem 2018;47:368-377 $\begin{array}{lll}\text { DOI: 10.1159/000489917 } & \text { Ond Biochemistry } & \begin{array}{l}\text { 2018 The Author(s). Published by S. Karger AG, Basel } \\ \text { www.karger.com/cpb }\end{array}\end{array}$ Zacharopoulou et al.: KDM2B Regulates EMT and Rho Signaling in Colon Cancer Cells}

\section{Introduction}

The epigenetic factor KDM2B is a novel oncogene, coding for a lysine-specific demethylase that removes $\mathrm{H} 3 \mathrm{~K} 4 \mathrm{me} 3$ and $\mathrm{H} 3 \mathrm{~K} 36 \mathrm{me} 2$ histone marks from the histones leading to transcriptional repression [1-4]. The function of KDM2B depends on the co-operation with Polycomb Repressor Complex-2 (PRC-2) that enables MEFs to undergo immortalization [4]. KDM2B is regulated by the basic fibroblast growth factor (bFGF) in human tumors that in turn governs various tumor characteristics including cell growth, migration, invasiveness, angiogenesis and actin cytoskeleton organization [5-7]. A previous study reported control of cell migration and angiogenesis by KDM2B in HUVECs [6]. In line with this report, recent studies from our group in DU-145 prostate cancer cells established a significant role of this epigenetic factor in regulating cell migration [8]. Analysis of the underling mechanism revealed the involvement of actin cytoskeleton reorganization, Rho GTPase signaling and cell-cell adhesion molecules E-cadherin and ZO-1 [8]. These findings supported previous reports suggesting that epigenetic mechanisms regulate actin cytoskeleton architecture [8-10] that in turn is a crucial mediator conferring cancer cells with migratory and invasive characteristics [11-21]. In addition, these studies established a clear link between KDM2B function, actin architecture and migration in prostate cancer cells [8]. However, it remained unclear whether the control of actin cytoskeleton signaling mechanisms, including activity of Rho GTPases, by this epigenetic factor is a general regulatory phenomenon, relevant for other tumor cells and whether KDM2B-induced cell-cell adhesion alterations may as well influence epithelial to mesenchymal transition (EMT). To this end, in the present study we addressed whether epigenetic alterations result in the deregulation of small GTPases function and may influence EMT in colon tumor cells. For this, we analyzed whether KDM2B governs the expression of small GTPases genes, including RhoA/RhoB/RhoC and Rac1, and addressed its role in regulating the EMT markers E-cadherin, N-cadherin and ZO-1 in HCT116 colon cancer cells. Our findings fully support the notion that in tumor cells, epigenetic mechanisms control the expression and function of EMT and cytoskeletal genes.

\section{Materials and Methods}

RPMI 1640 medium, penicillin/streptomycin, trypsin, puromycin, SuperScript II Reverse Transcriptase, Trizol reagent for RNA extraction, dNTPs, ProLong Gold Antifade reagent with Dapi and Anti-Zo1 (339100) were purchased from Invitrogen/Life Technologies (Carlsbad, CA, USA). Fetal Bovine Serum (FBS) was purchased from Biochrom (Berlin, Germany), RT-PCR primers from Eurofins MWG Operon (Ebersberg, Germany). FITC-IgG antibody was from Molecular probes Inc (Eugene OR USA). Anti-Ezh2 (D2C9), anti-Bmi1 (D20B7), anti- N-Cadherin (13A9) and anti-RhoC (D40E4) antibodies were from Cell Signaling Technology (Danvers, MA, USA). Anti-RhoA (sc-418), anti-RhoB (sc-180) and anti-Rac1 (sc-95) were purchased from Santa Cruz Biotechnology Inc. (Santa Cruz, CA, USA) and KAPA SYBR Green/ROX qPCR Master Mix from Fermentas (Maryland, USA). Anti- JHDM1B (KDM2B) (09-864) antibody, Goat anti-rabbit and anti-mouse IgG Peroxidase Conjugated antibodies and ECL Immobilon western-Chemiluminescent HRP Substrate were purchased from Millipore Corporation (Billerica, MA, USA). Albumin Fraction V (BSA) was from PanReac Applichem, ITW Reagents (Gatersleben, Germany). All other chemicals were from usual commercial sources at the purest grade available.

Cell cultures, Lentiviral and Retroviral packaging and transduction

HCT116 and DU-145 cell lines were cultured in RPMI 1640, supplemented with 1\% penicillin/ streptomycin and $10 \%$ heat-inactivated fetal bovine serum in a humidified atmosphere containing $5 \% \mathrm{CO}_{2}$ at $37^{\circ} \mathrm{C}$. Lentiviral and retroviral packaging and transduction in HCT116 cells were performed as described previously [8]. Cells infected with retrovirus ((pBABEpuro-KDM2B (for overexpression KDM2B) or control empty vector pBABEpuro (control for overexpressing KDM2B cells)) or lentivirus (pLKO.1KDM2BshRNA (expressing either shKDM2B (pLKO.1-KDM2B-sh) or scramble shRNA (pLKO.1-scrambled-sh)) constructs. Lentiviral-based shRNAs for human KDM2B were purchased from Open Biosystems (cat no. RH54533- 


\section{Cellular Physiology Cell Physiol Biochem 2018;47:368-377 \begin{tabular}{ll|l} 
DOI: 10.1159/000489917 & Ond Biochemistry & $\begin{array}{l}\text { 2018 The Author(s). Published by S. Karger AG, Basel } \\
\text { www.karger.com/cpb }\end{array}$
\end{tabular} \\ Zacharopoulou et al.: KDM2B Regulates EMT and Rho Signaling in Colon Cancer Cells}

NM_001005366). Out of the 5 clones, the most effective in knocking down KDM2B was TRCN0000118437. Target Sequence: CGGCCTTTACAAGAAGACATT; Hairpin Sequence:5'-CCGG-CGGCCTTTACAAGAAGACATTCTCGAG AATGTCTTCTTGTAAAGGCCG-TTTTTG-3'. The lentiviral pLKO.1 vector, expressing a scrambled shRNA and the pLKO.1 retroviral empty vector (pBABEpuro) served as negative controls.

\section{Protein extraction and Western Blotting}

For Western blotting, lentiviral or retroviral transduced cells were lysed in lysis buffer: (Tris- $\mathrm{HCl} 50 \mathrm{mM}$ pH7.5, NaCl 150mM, Triton X-100 1\%, SDS 0, 2\% and sodium deoxycholate 0, 1\%, Protease/Phosphatase inhibitor cocktail of NaF 1M, Leupeptin 1M, $\beta$-glycerophosphate 10mM, Na3VO4 10mM, Aprotinin $80 \mu \mathrm{M}$, Pepstatin A 1mM, E-64 1, 5mM, Bestatin 5mM, AEBSF 100mM). Total protein extracts were analyzed by Western blotting followed by nitrocellulose membrane blotting with specific antibodies, as previously described [8]. Protein band intensity was quantified by using Image Lab Software (Bio-Rad).

RNA isolation, cDNA synthesis and Real time PCR

The cell lysates were processed for total RNA extraction using Trizol reagent according to the manufacturer's instructions. cDNA synthesis and real time PCR were performed as described previously [8]. For the amplification the following primers were used (5'-3' orientation):

Actin, forward CGGCATCGTCACCAACTG;

Actin, reverse GGCACACGCAGCTCATTG;

Kdm2b, forward TCTACGAGATCGAGGACAGGA;

Kdm2b, reverse ACCAGCACATCTCATAGTAGAAGG .

\section{Immunofluorescence}

Immunofluorescence microscopy experiments in HCT116 cells were performed as described previously [8]. Primary antibodies used were anti-JHDM1B (KDM2B) (1:100, 09-864, Millipore), anti-EZH2 (1:100, D2C9, Cell Signaling Technology) and anti-BMI 1 (1:100, D20B7, Cell Signaling Technology), and secondary antibodies Alexa 555-conjugated goat (1:600, anti-rabbit, Invitrogen) and FITC 488 (1:500, anti-mouse, Invitrogen). The slides were mounted using the ProLong Gold Antifade reagent with Dapi. Photomicrographs were obtained with a LEICA microscope (LEICA DM LB) and were photographed with a LEICA DC 300F digital camera, using the Zeiss Plan-neofluar $40 \times / 0.75$ objective lens. The images were analyzed with the instrument's software.

\section{Replicates and Statistical Analysis}

All experiments were performed in 3 independent replications and data are presented as means \pm standard deviation (SD). All data were tested for significance using unpaired Student t-test, with $\mathrm{P}$ values of 0.05 or less being considered statistically significant $\left({ }^{*}(\mathrm{p}<0.05),{ }^{* *}(\mathrm{p}<0.01),{ }^{* * *}(\mathrm{p}<0.001)\right)$.

\section{Results}

In line with previous reports [8], expression of KDM2B protein was evident in HCT116 colon tumor cells (Fig. 1A). Compared to scramble shRNA, knockdown of KDM2B revealed downregulation of both, gene transcripts (Fig. 1B, left panel) and protein levels (Fig. 1C, left panels). On the other hand, overexpression of KDM2B bare up-regulation of gene transcripts (Fig. 1B, right panel) and protein levels (Fig. 1C, right panels). These findings were fully supported by IF microscopic analysis (Fig. 1D).

KDM2B gene was functional in HCT116 cells, since knockdown resulted in clear reduction of EZH2 protein levels (Fig. 2A, left panels), whereas overexpression induced enhanced EZH2 expression (Fig. 2A, right panel). In addition to EZH2, KDM2B regulated as well BMI1 protein levels in HCT116 cells (Fig. 2B). IF microscopic observations (Fig. 2C and D) strengthened these findings.

We further analyzed the role of KDM2B in controlling EMT in tumor cells by assessing the expression profiles of the EMT markers E-Cadherin, ZO-1 and N-Cadherin. Knockdown of KDM2B gene in HCT116 cell clones revealed elevated protein expression of the epithelial 


\section{Cellular Physiology and Biochemistry

Fig. 1. Expression of KDM2B in tumor cells. (A) Protein expression of KDM2B and tubulin (control) in HCT116 and DU145 cell lysates. Western blot analysis established differential basic KDM2B expression levels. (B) KDM2B mRNA levels were measured by quantitative real time RT-PCR, with $\beta$-actin as the internal control in scramble shRNA, shKDM2B (left panel), and control overexpression, overexpression of KDM2B (right panel) HCT116 cell lysates. The data are presented as mean \pm standard deviation (SD) from $n=3$ independent experiments, while y axis represents the ratio between KDM2B and actin genes; ${ }^{* * *} \mathrm{p}<0.001$ indicates statistical significance. (C) Original Western blot and quantification of the expression of KDM2B and tubulin (control) protein in scramble shRNA, shKDM2B (left panel) and control overexpression, overexpression of KDM2B (right panel) HCT116 cell lysates. Graph shows mean expression of KDM2B relative to control from $n=3$ independent experiments; ${ }^{* * *} \mathrm{p}<0.001$ indicates statistical significance. (D) Immunofluorescence of KDM2B (red) staining and nuclei (blue) in control (left), shKDM2B (middle) and overexpression of KDM2B (right) HCT116 cells. Scale bar represents $100 \mu \mathrm{m}$.
Fig. 2. KDM2B controls the expression of EZH2 and BMI1 in HCT116 colon cancer cells. (A) Original Western blot and quantification of the expression of EZH2 and tubulin protein in scramble shRNA, shKDM2B (left panel) and control overexpression, overexpression of KDM2B (right panel) HCT116 cell lysates. Graph shows mean expression of EZH2 relative to control from $n=3$ independent experiments; ${ }^{* * *} \mathrm{p}<0.001$ indicates statistical significance. (B) Original Western blot and quantification of the expression of BMI 1 and tubulin protein in scramble shRNA, shKDM2B (left panel) and control overexpression, overexpression of KDM2B (right panel) HCT116 cell lysates. Graph shows mean expression of BMI 1 relative to control from n=3 independent experiments; ${ }^{*}(\mathrm{p}<0.05) ;{ }^{* *}(\mathrm{p}<0.01)$ indicates statistical significance. (C) Immunofluorescence of EZH2 (red) staining and nuclei (blue) in control (left), shKDM2B (middle) and overexpression of KDM2B (right) HCT116 cells. Scale bar represents $100 \mu \mathrm{m}$. (D) Immunofluorescence of BMI1 (red) staining and nuclei (blue) in control (left), shKDM2B (middle) and overexpression of KDM2B (right) HCT116 cells. Scale bar represents $100 \mu \mathrm{m}$. c
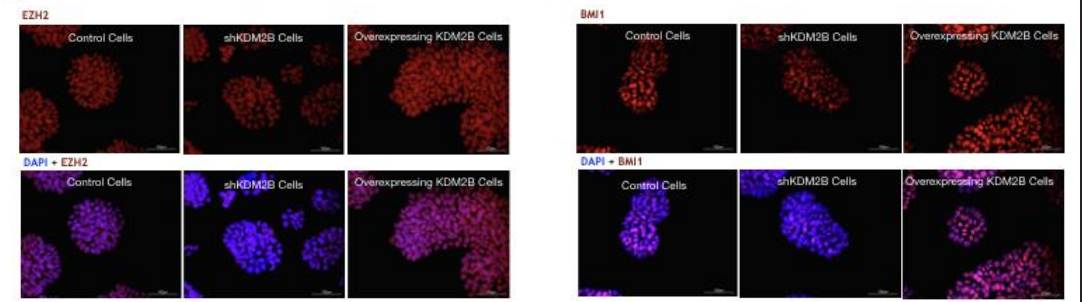

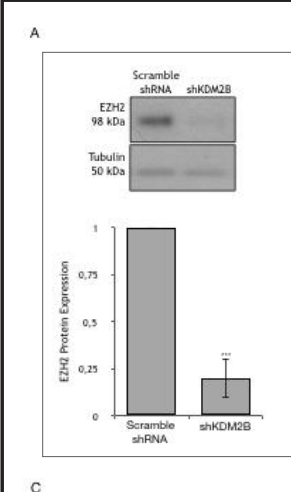




\section{Cellular Physiology and Biochemistry

Fig. 3. KDM2B controls the expression of E-Cadherin, ZO-1 and $\mathrm{N}$-Cadherin. (A) Original Western blot and quantification of the expression of E-Cadherin and tubulin protein in scramble shRNA, shKDM2B (left panel) and control overexpression, overexpression of KDM2B (right panel) HCT116 cell lysates. Graph shows mean expression of E-Cadherin relative to control from $n=3$ independent experiments; ${ }^{*}(\mathrm{p}<0.05)$ indicates statistical significance. (B) Original Western blot and quantification of the expression of ZO-1 and tubulin protein in scramble shRNA, shKDM2B (left panel) and control overexpression, overexpression of KDM2B (right panel)
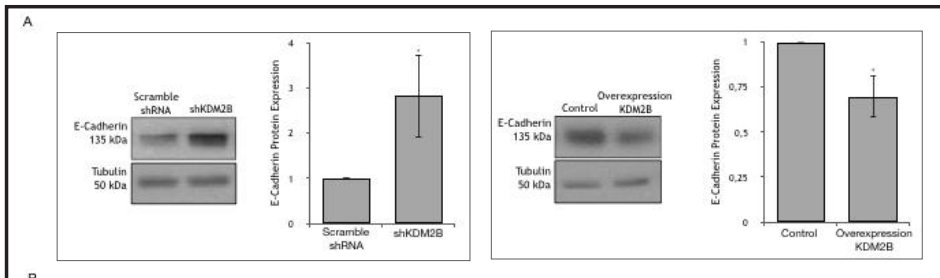

$$
\text { B }
$$
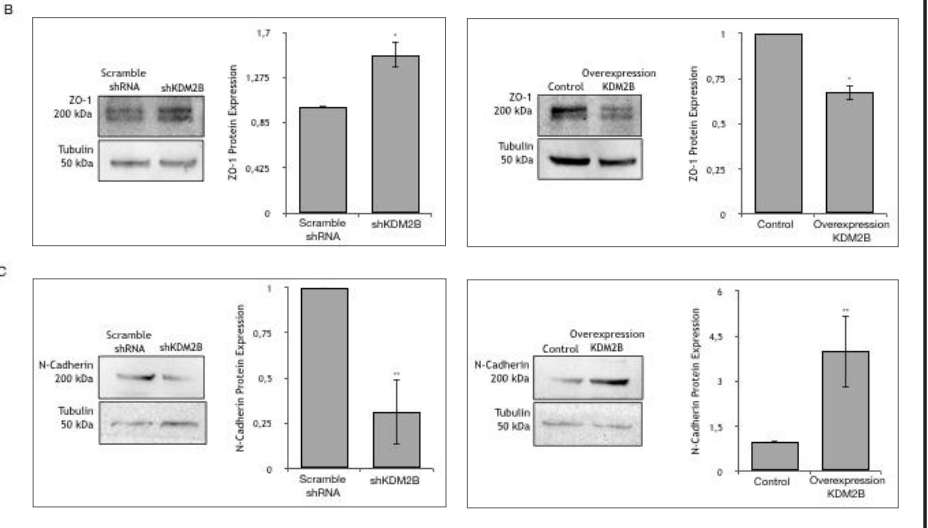

HCT116 cell lysates. Graph shows mean expression of $\mathrm{ZO}-1$ relative to control from $\mathrm{n}=3$ independent experiments; ${ }^{*}(\mathrm{p}<0.05)$ indicates statistical significance. (C) Original Western blot and quantification of the expression of $\mathrm{N}$-Cadherin and tubulin protein in scramble shRNA, shKDM2B (left panel) and control overexpression, overexpression of KDM2B (right panel) HCT116 cell lysates. Graph shows mean expression of N-Cadherin relative to control from n=3 independent experiments; ${ }^{* *}(\mathrm{p}<0.01)$ indicates statistical significance.

Fig. 4. KDM2B regulates expression of small Rho GTPases. (A) Original Western blot and quantification of the expression of RhoA and tubulin protein in scramble shRNA, shKDM2B (left panel) and control overexpression, overexpression of KDM2B (right panel) HCT116 cell lysates. Graph shows mean expression of RhoA relative to control from $n=3$ independent experiments; $*(p<0.05)$ indicates statistical significance. (B) Original Western blot and quantification of the expression of RhoB and tubulin protein in scramble shRNA, shKDM2B (left panel) and control overexpression, overexpression of KDM2B (right panel) HCT116 cell lysates. Graph shows
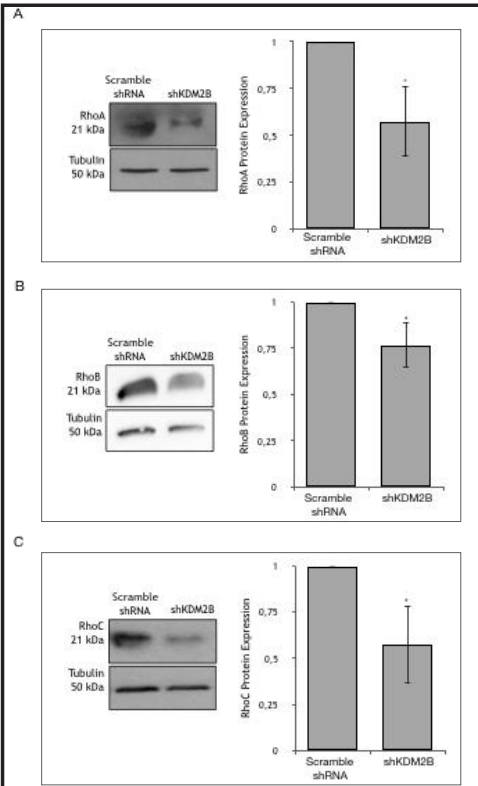
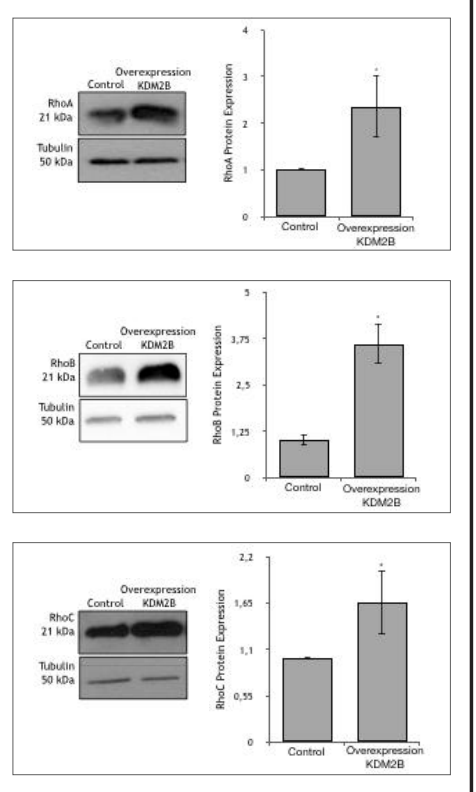
mean expression of RhoB relative to control from $n=3$ independent experiments; ${ }^{*}(\mathrm{p}<0.05)$ indicates statistical significance. (C) Original Western blot and quantification of the expression of RhoC and tubulin protein in scramble shRNA, shKDM2B (left panel) and control overexpression, overexpression of KDM2B (right panel) HCT116 cell lysates. Graph shows mean expression of RhoC relative to control from $n=3$ independent experiments; ${ }^{*}(p<0.05)$ indicates statistical significance. 
Fig. 5. KDM2B regulates expression of Rac1 small GTPase in HCT116 colon tumor- and DU-145 prostate cancer- cells. (A) Original Western blot and quantification of the expression of Rac1 and tubulin protein in scramble shRNA, shKDM2B (left panel) and control overexpression, overexpression of KDM2B (right pa-

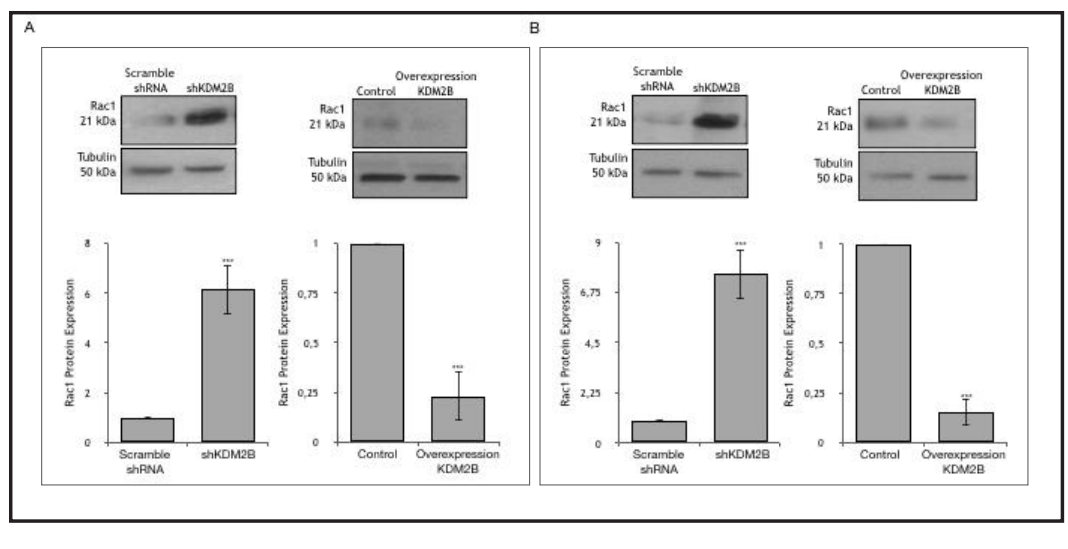
nel) HCT116 cell lysates.

Graph shows mean expression of Rac1 relative to control from $\mathrm{n}=3$ independent experiments; ${ }^{* * *} \mathrm{p}<0.001$ indicates statistical significance. (B) Original Western blot and quantification of the expression of Rac1 and tubulin protein in scramble shRNA, shKDM2B (left panel) and control overexpression, overexpression of KDM2B (right panel) DU-45 cell lysates. Graph shows mean expression of Rac1 relative to control from $\mathrm{n}=3$ independent experiments; ${ }^{* * *} \mathrm{p}<0.001$ indicates statistical significance.

marker E-Cadherin (Fig. 3A, left panels), while cell clones overexpressing KDM2B showed reduced E-Cadherin protein levels (Fig. 3A, right panels). These findings are in line with recently reported regulation of E-Cadherin gene transcription by KDM2B in DU-145 cells [8]. Very similar results were obtained for an additional epithelial marker, namely ZO-1 (Fig. 3B). Finally, we assessed the expression profile of the mesenchymal marker N-Cadherin. Interestingly, shRNA-mediated knockdown of KDM2B in HCT116 cell clones revealed potent downregulation of protein expression (Fig. 3C, left panels), while cell clones overexpressing KDM2B showed enhanced N-Cadherin protein levels (Fig. 3C, right panels). These results suggest that the epigenetic factor KDM2B regulates EMT in HCT116 colon tumor cells.

In our recent study [8], we demonstrated a clear correlation between KDM2B function, actin organization and small GTPases signaling in prostate cancer cells. Moreover, previous reports established a functional link between EMT, actin cytoskeleton reorganization and small GTPases signaling [20-27]. Accordingly, we further analyzed the functional link of the KDM2B gene with various small GTPase family proteins, namely RhoA/B/C and Rac1 in colon tumor cells. Knockdown of KDM2B in HCT116 cell clones revealed a clear decrease of RhoA, RhoB and RhoC protein levels as manifested by Western blotting (Fig. 4A, B, C, left panels), while overexpression of KDM2B exhibited enhanced protein levels of all tested small GTPases (Fig. 4A, B, C, right panels). Interestingly, the opposite effect was evident for Rac1. As shown in Fig. 5A, clear upregulation of the Rac1 small GTPase protein expression became evident upon knockdown of KDM2B in HCT116 cell clones (Fig. 5A, left panels), while KDM2B overexpression induced downregulation of Rac1 protein level (Fig. 5A, right panels). We further investigated whether this finding was evident as well in other tumor cells upon KDM2B knockdown or overexpression. We observed very similar results in DU145 prostate cancer cells, since knockdown or overexpression respectively of KDM2B in these cells revealed clear enhanced (Fig. 5B left panel) or diminished (Fig. 5B right panel) Rac1 protein expression.

\section{Discussion}

In this study, we addressed the regulatory role of the epigenetic factor KDM2B by analyzing EMT and Rho GTPases in HCT116 colon tumor cells. Our results established that KDM2B overexpression or knockdown regulates: a) the expression profiles of EZH2 and 


\section{Cellular Physiology Cell Physiol Biochem 2018;47:368-377

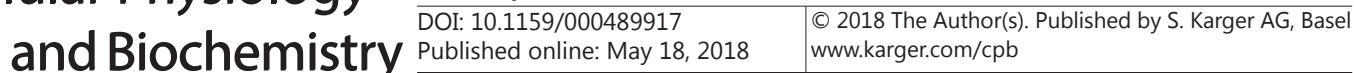 \\ Zacharopoulou et al.: KDM2B Regulates EMT and Rho Signaling in Colon Cancer Cells}

BMI1, b) the expression levels of the epithelial markers E-Cadherin and ZO-1, as well as the mesenchymal marker N-Cadherin, c) the protein levels of RhoA, RhoB and RhoC small GTPases and finally, d) the protein levels of Rac1 small GTPase. These results imply that this epigenetic factor controls EMT and small Rho GTPase expression in HCT116 colon cancer cells and is in line with previously reported findings in prostate cancer cells [8]. Taking together, these results argue that KDM2B has a significant oncogenic role in regulating cell functions in various tumors.

Previous work showed that the upregulation of KDM2B in co-operation with Polycomb group proteins promotes motility and invasion in tumor cell lines and primary tumors $[5,6]$. In line with these reports, in a recent study from our group we elucidated a novel signaling pathway that is regulating KDM2B-induced migration in prostate tumor cells [8]. This mechanism involved the small GTPases RhoA and RhoB, the epithelial markers E-Cadherin and ZO-1 as well as deregulation of actin cytoskeleton organization, which in turn controlled the migration of prostate tumor cells [8]. Interestingly, as previously reported, a migration assay failed to establish a clear relationship between KDM2B expression and motility in HCT116 colon tumor cells [8]. Since KDM2B protein levels in HCT116 cells are significantly lower compared to DU-145 prostate cancer cells ([8], and Fig. 1A), this observation suggests that variable basal KDM2B expression levels may differentially regulate the migratory behavior of tumor cells and needs further investigation.

Our data establish also a clear involvement of epigenetic factors in controlling EMT. This became evident from the opposite effects on the expression of the epithelial markers E-Cadherin and ZO-1 versus the mesenchymal marker N-Cadherin, shown upon knockdown or overexpression of the KDM2B gene. This observation implies a significant role of KDM2B in tumorigenesis and suggests the implication of actin cytoskeleton and small GTPases signaling in this phenomenon. Indeed, this assumption was postulated previously in various tumor cell models by analyzing the correlation of EMT with actin signaling and migration $[13,19-27]$. Moreover, previous studies showed that the Serum glucocorticoid kinase 1 (Sgk1) plays a key role in EMT in colon carcinoma [28]. Furthermore, Sgk1 regulates epigenomic re-alignment and drug-resistance through the control of GTP-proteins RANBP1 and RAN [29]. Accordingly, co-regulation between KDM2B and Sgk1 seems possible and needs further investigation.

Regulation of Rho small GTPases function is a crucial step in the oncogenic behavior of various tumor cells, including growth, migration and apoptosis [11, 15, 16, 18, 30, 31]. Rho signaling governs actin redistribution [32-41] and is implicated in various cellular responses including cell-cell adhesion and EMT $[13,22,25,42-45]$. In the present work, we reported opposite effects of the KDM2B gene in the expression of RhoA/RhoB/RhoC- and Rac1GTPases. This is an interesting observation that needs further analysis. However, since Rho GTPases are implicated in the formation of actin stress fibers [46, 47], while Rac1 promotes restructuring of actin cytoskeleton towards lamelipodia [47-50] our findings may indicate that the epigenetic factor KDM2B plays a differential role in actin reorganization. This may include promotion of lamelipodia formation with concomitant inhibition of stress fibers and vice versa. Such a mechanism may be crucial for controlling simultaneously both, growth and motility of tumor cells [48, 51-53]. In addition, since KDM2B governed Rac1 regulation was as well evident in DU-145 prostate cancer cells (Fig. 5B), we assume that this regulatory step may as well contribute to the reported control of cell migration of prostate cancer cells [8], as previously described in breast cancer cells [54]. On the other hand, regulation of Rac1 GTPase expression in HCT116 cell may be correlated with the control of tumor cells invasion from the intestine epithelium into the endothelium of blood vessels through the formation of lamelipodia, rather than controlling the migration in wound healing experiments in a flat substrate such as cell culture flask. Additional work is now needed to address these interesting issues.

To conclude, in the present work we demonstrate that the histone demethylase KDM2B is regulating the expression of EMT markers E-Cadherin, ZO-1 and N-Cadherin and the small GTPases RhoA/B/C and Rac1 in colon tumor cells. Taking into consideration the recently 


\section{Cellular Physiology Cell Physiol Biochem 2018;47:368-377 \begin{tabular}{l|l|l} 
DOI: 10.1159/000489917 & O 2018 The Author(s). Published by S. Karger AG, Basel \\
www.karger.com/cpb
\end{tabular} \\ Zacharopoulou et al.: KDM2B Regulates EMT and Rho Signaling in Colon Cancer Cells}

shown effects of KDM2B in prostate cancer cells [8], these interactions imply a direct involvement of this epigenetic factor in EMT and deregulation of cytoskeletal genes that may govern oncogenic behavior of tumor cells.

\section{Acknowledgements}

Authors appreciate the funding of this work by the International Scientific Partnership Program ISPP at the King Saud University through ISPP\#009, the University of Crete Research Committee. We acknowledge support by Deutsche Forschungsgemeinschaft and Open Access Publishing Fund of University of Tübingen

\section{Disclosure Statement}

The authors declare that they do not have any conflict of interests.

\section{References}

1 He J, Nguyen AT, Zhang Y: KDM2b/JHDM1b, an H3K36me2-specific demethylase, is required for initiation and maintenance of acute myeloid leukemia. Blood 2011;117:3869-3880.

-2 Tzatsos A, Paskaleva P, Lymperi S, Contino G, Stoykova S, Chen Z, Wong KK, Bardeesy N: Lysine-specific demethylase $2 \mathrm{~b}$ (KDM2B)-let-7-enhancer of zester homolog 2 (EZH2) pathway regulates cell cycle progression and senescence in primary cells. J Biol Chem 2011;286:33061-33069.

-3 Kampranis SC, Tsichlis PN: Chapter 4 Histone Demethylases and Cancer. Adv Cancer Res 2009;102:103169.

-4 Tzatsos A, Pfau R, Kampranis SC, Tsichlis PN: Ndy1/KDM2B immortalizes mouse embryonic fibroblasts by repressing the Ink4a/Arf locus. Proc Natl Acad Sci U S A 2009;106:2641-2646.

5 Kottakis F, Foltopoulou P, Sanidas I, Keller P, Wronski A, Dake BT, Ezell SA, Shen Z, Naber SP, Hinds PW, McNiel E, Kuperwasser C, Tsichlis PN: NDY1/KDM2B functions as a master regulator of polycomb complexes and controls self-renewal of breast cancer stem cells. Cancer Res 2014;74:3935-3946.

6 Kottakis F, Polytarchou C, Foltopoulou P, Sanidas I, Kampranis SC, Tsichlis PN: FGF-2 Regulates Cell Proliferation, Migration, and Angiogenesis through an NDY1/KDM2B-miR-101-EZH2 Pathway. Mol Cell 2011;43:285-298.

7 Fajol A, Honisch S, Zhang B, Schmidt S, Alkahtani S, Alarifi S, Lang F, Stournaras C, Föller M: Fibroblast growth factor (Fgf) 23 gene transcription depends on actin cytoskeleton reorganization. FEBS Lett 2016;590:705-715.

8 Zacharopoulou N, Tsapara A, Kallergi G, Schmid E, Tsichlis PN, Kampranis SC, Stournaras C: The epigenetic factor KDM2B regulates cell adhesion, small rho GTPases, actin cytoskeleton and migration in prostate cancer cells. Biochim Biophys Acta - Mol Cell Res 2018;1865:587-597.

-9 Gari HH, DeGala GD, Ray R, Lucia MS, Lambert JR: PRL-3 engages the focal adhesion pathway in triplenegative breast cancer cells to alter actin structure and substrate adhesion properties critical for cell migration and invasion. Cancer Lett 2016;380:505-512.

10 Wang P, Zhao J, Yang X, Guan S, Feng H, Han D, Lu J, Ou B, Jin R, Sun J, Zong Y, Feng B, Ma J, Lu A, Zheng M: PFDN1, an indicator for colorectal cancer prognosis, enhances tumor cell proliferation and motility through cytoskeletal reorganization. Med Oncol 2015;32:1-13.

11 Vega FM, Ridley AJ: The RhoB small GTPase in physiology and disease. Small GTPases 2016;0:1-10.

-12 Callan-Jones AC, Voituriez R: Actin flows in cell migration: From locomotion and polarity to trajectories. Curr Opin Cell Biol 2016;38:12-17.

13 Vega FM, Thomas M, Reymond N, Ridley AJ: The Rho GTPase RhoB regulates cadherin expression and epithelial cell-cell interaction. Cell Commun Signal 2015;13:1-9.

14 Insall RH, Machesky LM: Actin Dynamics at the Leading Edge: From Simple Machinery to Complex Networks. Dev Cell 2009;17:310-322. 


\section{Cellular Physiology Cell Physiol Biochem 2018;47:368-377 \begin{tabular}{ll|l} 
DOI: 10.1159/000489917 & O 2018 The Author(s). Published by S. Karger AG, Basel \\
www.karger.com/cpb
\end{tabular}

15 Papakonstanti EA, Stournaras C: Cell responses regulated by early reorganization of actin cytoskeleton. FEBS Lett 2008;582:2120-2127.

16 Stournaras C, Gravanis A, Margioris AN, Lang F: The actin cytoskeleton in rapid steroid hormone actions. Cytoskeleton 2014;71:285-293.

17 Vignjevic D, Montagnac G: Reorganisation of the dendritic actin network during cancer cell migration and invasion. Semin Cancer Biol 2008;18:12-22.

18 Tseliou M, Al-Qahtani A, Alarifi S, Alkahtani SH, Stournaras C, Sourvinos G: The Role of RhoA, RhoB and RhoC GTPases in Cell Morphology, Proliferation and Migration in Human Cytomegalovirus (HCMV) Infected Glioblastoma Cells. Cell Physiol Biochem 2016;38:94-109.

19 Lang F, Perrotti N, Stournaras C: Colorectal carcinoma cells-Regulation of survival and growth by SGK1 Int J Biochem Cell Biol 2010;42:1571-1575.

20 Honisch S, Yu W, Liu G, Alesutan I, Towhid ST, Tsapara A, Schleicher S, Handgretinger R, Stournaras C, Lang F: Chorein addiction in VPS13A overexpressing rhabdomyosarcoma cells. Oncotarget 2015;6:1030910319.

21 Gu S, Papadopoulou N, Nasir O, Föller M, Alevizopoulos K, Lang F, Stournaras C: Activation of Membrane Androgen Receptors in Colon Cancer Inhibits the Prosurvival Signals Akt/Bad In vitro and In vivo and Blocks Migration via Vinculin/Actin Signaling. Mol Med 2011;17:48-58.

22 Papadimitriou E, Vasilaki E, Vorvis C, Iliopoulos D, Moustakas a, Kardassis D, Stournaras C: Differential regulation of the two RhoA-specific GEF isoforms Net1/Net1A by TGF- $\beta$ and miR-24: role in epithelial-tomesenchymal transition. Oncogene 2011;31:18-21.

23 Schneider D, Janshoff A: Inhibition of actin dynamics during epithelial-to-mesenchymal transition. Biochem Biophys Res Commun 2012;419:221-225.

24 Zhang L, Jiang G, Zhao X, Gong Y: Dimethyloxalylglycine Promotes Bone Marrow Mesenchymal Stem Cell Osteogenesis via Rho/ROCK Signaling. Cell Physiol Biochem 2016;39:1391-1403.

25 Niu H, Wu B, Jiang H, Li H, Zhang Y, Peng Y, He P: Mechanisms of RhoGDI2 mediated lung cancer epithelialmesenchymal transition suppression. Cell Physiol Biochem 2014;34:2007-2016.

26 Ju L, Zhou Z, Jiang B, Lou Y, Guo X: Autocrine VEGF and IL-8 promote migration via Src/Vav2/Rac1/PAK1 signaling in human umbilical vein endothelial cells. Cell Physiol Biochem 2017;41:1346-1359.

27 Ungefroren H, Witte D, Lehnert H: The role of small GTPases of the Rho/Rac family in TGF- $\beta$-induced EMT and cell motility in cancer. Dev Dyn 2017;doi: 10.1002/dvdy.24505 [Epub ahead of print]. Rev.

28 Talarico C, Dattilo V, D’Antona L, Menniti M, Bianco C, Ortuso F, Alcaro S, Schenone S, Perrotti N, Amato R: SGK1, the New Player in the Game of Resistance: Chemo-Radio Molecular Target and Strategy for Inhibition. Cell Physiol Biochem 2016;39:1863-1876.

29 Dattilo V, D’Antona L, Talarico C, Capula M, Catalogna G, Iuliano R, Schenone S, Roperto S, Cataldo B, Perrotti N, Amato R: SGK1 affects RAN/RANBP1/RANGAP1 via SP1 to play a critical role in pre-miRNA nuclear export: A new route of epigenomic regulation. Sci Rep 2017;7:1-14.

-30 Zandvakili I, Lin Y, Morris JC, Zheng Y: Rho GTPases: Anti- or pro-neoplastic targets? Oncogene 2017;36:3213-3222.

-31 O'Connor KL, Chen M: Dynamic functions of RhoA in tumor cell migration and invasion. Small GTPases 2013;4:141-147.

32 Koukouritaki SB, Gravanis A, Stournaras C: Tyrosine phosphorylation of focal adhesion kinase and paxillin regulates the signaling mechanism of the rapid nongenomic action of dexamethasone on actin cytoskeleton. Mol Med 1999;5:731-42.

-33 Liu D, Xiong R, Chen X, Li P, Ning Y, Peng Y, Zhao Y, Yang N, Zhou Y: The glucocorticoid dexamethasone inhibits U937 cell adhesion and neutrophil release via RhoA/ROCK1-dependent and independent pathways. Cell Physiol Biochem 2014;33:1654-1662.

-34 Koukouritaki SB, Margioris AN, Gravanis A, Hartig R, Stournaras C: Dexamethasone Induces Rapid Actin Assembly in H uman Endometrial Cells W ithout Affecting Its Synthesis. J Cell Biochem 1997;65:492-500.

-35 Gu S, Papadopoulou N, Gehring EM, Nasir O, Dimas K, Bhavsar SK, Föller M, Alevizopoulos K, Lang F, Stournaras C: Functional membrane androgen receptors in colon tumors trigger pro-apoptotic responses in vitro and reduce drastically tumor incidence in vivo. Mol Cancer 2009;8:1-14.

-36 Vardouli L, Vasilaki E, Papadimitriou E, Kardassis D, Stournaras C: A novel mechanism of TGF $\beta$-induced actin reorganization mediated by Smad proteins and Rho GTPases. FEBS J 2008;275:4074-4087. 


\section{Cellular Physiology Cell Physiol Biochem 2018;47:368-377

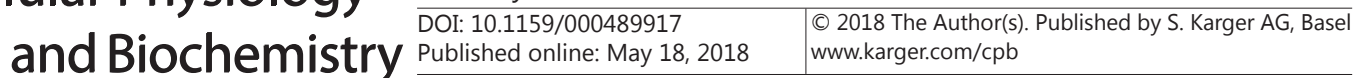 \\ Zacharopoulou et al.: KDM2B Regulates EMT and Rho Signaling in Colon Cancer Cells}

37 Nomikou E, Stournaras C, Kardassis D: Functional analysis of the promoters of the small GTPases RhoA and RhoB in embryonic stem cells. Biochem Biophys Res Commun 2017;491:754-759.

-38 Schmid E, Gu S, Yang W, Münzer P, Schaller M, Lang F, Stournaras C, Shumilina E: Serum- and glucocorticoid-inducible kinase SGK1 regulates reorganization of actin cytoskeleton in mast cells upon degranulation. AJP Cell Physiol 2013;304:C49-55.

-39 Gu S, Kounenidakis M, Schmidt EM, Deshpande D, Alkahtani S, Alarifi S, Föller M, Alevizopoulos K, Lang F, Stournaras C: Rapid activation of FAK/mTOR/p70S6K/PAK1-signaling controls the early testosteroneinduced actin reorganization in colon cancer cells. Cell Signal 2013;25:66-73.

-40 Kardassis D, Murphy C, Fotsis T, Moustakas A, Stournaras C: Control of transforming growth factor signal transduction by small GTPases. FEBS J 2009;276:2947-2965.

41 Papakonstanti EA, Vardaki EA, Stournaras C: Actin cytoskeleton: a signaling sensor in cell volume regulation. Cell Physiol Biochem 2000;10:257-64 Review.

42 Zeng N, Salker MS, Zhang S, Singh Y, Shi B, Stournaras C, Lang F: 1 $\alpha, 25(\mathrm{OH}) 2 \mathrm{D} 3$ Induces Actin Depolymerization in Endometrial Carcinoma Cells by Targeting RAC1 and PAK1 Cell Physiol Biochem 2016;40:1455-1464.

-43 Gu S, Honisch S, Kounenidakis M, Alkahtani S, Alarif S, Alevizopoulos K, Stournaras C, Lang F: Membrane androgen receptor down-regulates c-Src-activity and beta-catenin transcription and triggers GSK-3betaphosphorylation in colon tumor cells. Cell Physiol Biochem 2014;34:1402-1412.

-44 Alesutan I, Seifert J, Pakladok T, Rheinlaender J, Lebedeva A, Towhid ST, Stournaras C, Voelkl J, Schäffer TE, Lang F: Chorein sensitivity of actin polymerization, cell shape and mechanical stiffness of vascular endothelial cells. Cell Physiol Biochem 2013;32:728-742.

-45 Zou W, Meng X, Cai C, Zou M, Tang S, Chu X, Wang X, Zou F: Store-operated Ca2+ entry (SOCE) plays a role in the polarization of neutrophil-like HL-60 cells by regulating the activation of Akt, Src, and Rho family GTPases. Cell Physiol Biochem 2012;30:221-37.

46 Burridge K, Guilluy C: Focal adhesions, stress fibers and mechanical tension. Exp Cell Res 2016;343:14-20.

47 Parri M, Chiarugi P: Rac and Rho GTPases in cancer cell motility control. Cell Commun Signal 2010;8:1-14.

48 Fukata M, Nakagawa M, Kaibuchi K: Roles of Rho-family GTPases in cell polarisation and directional migration. Curr Opin Cell Biol 2003;15:590-597.

49 Kaverina I, Krylyshkina O, Small JV: Regulation of substrate adhesion dynamics during cell motility. Int J Biochem Cell Biol 2002;34:746-761.

50 Ridley AJ: Rho GTPase signalling in cell migration. Curr Opin Cell Biol 2015;36:103-112.

-51 Lang F, Alevizopoulos K, Stournaras C: Targeting membrane androgen receptors in tumors. Expert Opin Ther Targets 2013;17:951-963.

52 Linseman DA, Loucks FA: Diverse roles of Rho family GTPases in neuronal development, survival, and death. Front Biosci 2008;1:657-76.

-53 Papakonstanti EA, Stournaras C: Tumor necrosis factor-alpha promotes survival of opossum kidney cells via Cdc42-induced phospholipase C-gamma1 activation and actin filament redistribution. Mol Biol Cell 2004;15:1273-86.

54 Kallergi G, Agelaki S, Markomanolaki H, Georgoulias V, Stournaras C: Activation of FAK / PI3K / Rac1 Signaling Controls Actin Reorganization and Inhibits Cell Motility in Human Cancer Cells. Cell Physiol Biochem 2007;977-986. 